\title{
JACQUES ROUMAIN, ETHNOLOGUE HAÏTIEN
}

Christine Laurière (CNRS, IIAC-LAHIC)

A propos de: Jacques Roumain, Euvres complètes, édition critique établie par Léon-François Hoffmann (coord.), Madrid, Paris, Allca XX, Editions de l’Unesco, collection Archivos, 2003, 1690 pages.

Ce sont les Euvres complètes d'un auteur singulier, qu'il nous est donné de lire grâce à cette ambitieuse publication, soigneusement établie par Léon-François Hoffman. Mondialement renommé pour son roman paysan Gouverneurs de la rosée et son recueil de poèmes Bois d'ébène, figure emblématique des lettres haïtiennes, «contemporain capital », ainsi que le qualifie René Depestre dans son magnifique Liminaire, Jacques Roumain se garda bien d'être un écrivain prônant l'extraterritorialité de la littérature vis-à-vis du monde. Il ne fut pas pour autant un chantre de la négritude pour elle-même. De quelques phrases lumineuses, René Depestre explicite l'engagement de Roumain pour la cause noire et conclut ainsi : « le sérieux intellectuel de Jacques Roumain invite dans la rigueur les Noirs à rationaliser la mythologie raciale, à la transcender, et surtout à la dépasser dans l'âpre remontée vers la condition humaine universelle » (p.XXVII). Mais ici, davantage qu'à son œuvre littéraire, à laquelle justice ne pourrait être rendue par 
l'auteur de ces lignes, c'est plutôt son bref parcours dans l'ethnologie - sept courtes années - qui retiendra l'attention. Quelques documents inédits dont n'ont pas eu connaissance les éditeurs permettront d'ajouter une petite pierre à la reconstitution de son itinéraire ${ }^{1}$.

Né en 1907 dans une famille de l'élite terrienne et politique haïtienne - un de ses grands-pères fut président de la République -, Jacques Roumain ne s'est jamais complu oisivement dans son statut d'héritier et de patricien. De retour en 1927 sur sa terre natale, après une scolarité en Suisse et un détour par l'Espagne des corridas, il choisit de s'engager dans le combat patriotique contre l'occupation états-unienne de Haïti, conspuant férocement la bourgeoisie qui collabore et s'arrange de cet état de fait. Volontiers polémique, il collabore à plusieurs revues et signe des articles et pamphlets au titre cristallin : «Le Drapeau haïtien n'est pas une loque !», «Comment on traite les nègres aux Etats-Unis », «Un prêtre a le droit d'être soldat quand sa patrie est en danger », etc. Son œuvre littéraire est quant à elle marquée dans le début de ces années 30 par la publication des nombreux poèmes et de romans, dont les Fantoches et La montagne ensorcelée, ce dernier contribuant à asseoir sa notoriété en Haïti de par l'originalité du thème abordé pour la première fois dans un roman haïtien, la vie quotidienne des paysans dans les mornes.

Activiste politique, son statut de membre fondateur du parti communiste haïtien en juin 1934 et de secrétaire général lui vaut des démêlés avec le président SténioVincent,

\footnotetext{
${ }^{1}$ A juste titre, Léon-François Hoffmann prévient d'emblée dans sa note préliminaire qu' « il suffit souvent de donner au public des Euvres complètes pour qu'elles cessent de l'être: leur parution entraîne fréquemment l'émergence de nouveaux documents dont les éditeurs n'avaient pas eu connaissance » (p.XLVIII).
} 
qui l'envoie en prison et dépêche en même temps un mandat de dépôt contre «le nommé Marx Karl, un chef-agitateur d'origine germanique que le Komintern a infiltré en Haïti afin d'ourdir avec le citoyen Roumain Jacques une complot contre la Sûreté de l'Etat » (p.XXIII) ! René Depestre rappelle que le président Vincent, entraîné sur sa vertueuse lancée, en profita pour mettre «hors-la-loi une liste de dix mots du Petit Larousse Illustré, tenus pour obscènes et attentatoires à l'ordre public : capital, syndicat, cellule, parti, prolétariat, classe, communisme, ouvrier, grève, révolution. » (id.) Cette opposition au gouvernement conduisit Jacques Roumain d'ailleurs plusieurs fois en prison : entre décembre 1928 et juin 1936, il effectua quatre séjours sous les verrous pour une durée totale de trente deux mois. Sa santé y fut durement éprouvée, expliquant sans doute qu'il mourut si jeune, à l'âge de 37 ans.

Contraint à l'exil après sa libération en juin 1936, il s'installe à Bruxelles avant de se fixer à Paris à l'automne 1937. Il avait déjà séjourné dans la capitale en juillet de la même année, à l'occasion de la tenue du Congrès des Ecrivains pour la Défense de la Culture. A la fin de ce congrès, une conversation avec Léon Damas, guyanais et ethnologue, l'aurait décidé, selon ce dernier, à s'inscrire à l'Institut d'Ethnologie, «ce qu'il fit avec d'autant plus d'enthousiasme que Jacques Roumain, Haïtien de nationalité et nègre conscient, ne pouvait que mettre à profit, pour le plus grand bien de tous, les cours des professeurs Rivet et Mauss. $»^{2}$ René Depestre souligne l'originalité de ce parcours, à rebours de ce que l'on aurait pu attendre d'un militant communiste fidèle à la ligne du Komintern :

\footnotetext{
${ }^{2}$ Rapporté par Léonce Viaud dans la rubrique «Activités du Bureau d'Ethnologie » ( in Lorimer Denis (éd.), «Le Musée du Bureau d'Ethnologie d'Haïti », publication du Bureau d'Ethnologie, série II, $\mathrm{n}^{\circ} 10$, mars 1953 : 65). Léonce Viaud fait état des commentaires que Léon Damas, lors d'un séjour à Haïti, inscrivit dans le livre d'or de l'Institut d'Ethnologie, le 19 janvier 1953. Référence bibliographique non répertoriée dans les Euvres complètes.
} 
«Que fait-il à Paris ou à Bruxelles en ces temps de veillée d'armes que nazisme, fascisme, colonialisme, communisme - sans que se recoupent entre eux leurs forfaits - imposent aux humanités de 1937 ? A trente ans, Roumain reprend humblement les études à l'université. [...] On le voit naviguer à contre-courant. Pourquoi l'anthropologie à la place des «Questions du léninisme » ou du «Marxisme et la question coloniale »? Que pouvait-il attendre d'un savoir universitaire qui, en ces temps-là, était encore l'apanage d'un club fermé de gourous blancs ? [...] Roumain, en intellectuel imaginatif et libre, demande à la Sorbonne les outils conceptuels de l'aventure particulière qui rendrait possible la remontée à la lumière des millions de victimes de l'esclavage et de la colonisation » (p.XXV).

André-Marcel d'Ans, dans un long article au titre pertinent : « Jacques Roumain et la fascination de l'ethnologie » (pp.1378-1428), propose une lecture critique des trois textes ethnologiques de Roumain $^{3}$, ainsi que de ses articles sur la campagne anti-superstitieuse et du roman Gouverneurs de la rosée, en respectant la chronologie de son cheminement scientifique. Il commence donc par une évocation du champ anthropologique français puisque c'est à Paris que Jacques Roumain commença des études d'ethnologie, consacrant une première sous-partie à «Paul Rivet et la naissance en France d'une science ethnologique ». S'il pointe avec raison le retard de l'institutionnalisation de cette discipline en France et le rôle primordial que joua Paul Rivet dans ce processus dans les années 1920, plusieurs erreurs historiques viennent malheureusement parasiter une démonstration au demeurant intéressante. Retraçant le parcours de Paul Rivet, et son insertion à son retour d'Equateur en 1906 dans les institutions savantes dédiées à l'anthropologie, au premier rang desquels figurait la Société d'Anthropologie, il écrit : «Comme pour la plupart des savants de son époque, il ne faisait en effet pas de doute aux yeux de Paul Rivet qu'une relation devait forcément exister entre les caractères physiques (et singulièrement les indices céphalométriques) et le "degré d'évolution intellectuelle" des différentes "races" » (p.1380). C'est faire un

\footnotetext{
${ }^{3}$ «Contribution à l'étude de l'ethnobotanique précolombienne des Grandes Antilles », Bulletin du Bureau d'ethnologie de la République d'Haïti (BERH), février 1942; «Le sacrifice du tambour-Assôtô(r), publications du BERH, ${ }^{\circ} 1$, mars 1943 ; « L'outillage lithique des Ciboney d'Haïti », Bulletin du BERH, 2, 1943. Ces articles sont republiés dans les Euvres complètes pp.1111-1156.
} 
mauvais procès à celui qui a, au contraire, disqualifié l'anthropométrie dans sa recherche des caractères d'infériorité raciale, déniant tout lien de cause à effet entre une caractéristique anatomique et l'intelligence. Faut-il rappeler que Paul Rivet, en 1909, dans son mémoire sur les «Recherches sur le prognathisme », affirmait qu'il est «absolument illégitime de rechercher dans ces variations [des indices anthropométriques] une sorte de barème du degré d'évolution intellectuelle des diverses races $»^{4}$ ? Paul Rivet n'a pas reçu le prix Broca pour ses travaux sur le métissage et le prognathisme (p.1380) - en novembre 1908, précisons-le; ce prix récompensa ses travaux sur l'Equateur. Paul Rivet n'a rien publié à cette date sur le métissage, et son mémoire sur le prognathisme, qui signe au demeurant sa rupture d'avec la Société d'Anthropologie, ne paraîtra qu'en 1909-1910 dans une revue affiliée au Muséum national d'histoire naturelle, L'Anthropologie. Sa prise de distance a lieu bien avant 1920 et non pour des raisons politiques (p.1380), mais dans les années 1909-1911, culminant avec la création de l'Institut français d'anthropologie en 1911. Ce ne sont d'ailleurs pas uniquement ses convictions politiques d'homme de gauche qui l'ont éloigné de cette société savante, ce sont d'abord des raisons scientifiques dans la mesure où il estimait qu'il était totalement vain de vouloir établir une hiérarchie des différentes « races » et où il remettait en cause le magistère de l'anthropologie physique sur les autres sciences humaines. Ses travaux démontrent qu'il reste cependant tributaire de la rhétorique raciale, tant la notion de race est consubstantielle à la construction intellectuelle même de l'anthropologie de cette époque, mais il refuse son instrumentalisation comme alibi

\footnotetext{
${ }^{4}$ Paul Rivet, «Recherches sur le prognathisme », L'Anthropologie, XX, 1909 : 35-49, 175-187, et XXI, 1910 : 505-518, 637-666. Citation p.643. Sur la prise de distance de Paul Rivet vis-à-vis de la Société d'anthropologie, du paradigme anthropométrique et de la place accordée à l'anthropologie physique, cf. Christine Laurière, « Le prognathe et l'anthropologue », L'Homme, 163, 2002 : 195-204 ; Filippo Zerilli, Il lato oscurodell'etnologia. Ilcontributodell'antropologia naturalista al processo di istituzionalizzazionedeglistudietnologici in Francia, Roma, CISU, 1998.
} 
inégalitaire.

Paul Rivet est bien élu professeur d'anthropologie au Muséum en 1928, mais il ne rebaptise pas immédiatement sa chaire «Ethnologie des hommes actuels et des hommes fossiles » (p.1381). Cette nouvelle dénomination ne sera votée qu'en janvier 1936, lors d'une assemblée des professeurs du Muséum ${ }^{5}$. Le musée d'ethnographie du Trocadéro est bien rattaché à sa chaire dans la foulée, le 27 mars 1928, mais il n’y fait pas « aussitôt nommer en tant que sous-directeur son collaborateur direct, Georges Henri Rivière » (p.1381), car il ne le connaît pas encore. Les deux hommes ne se rencontreront que deux mois plus tard, en mai 1928, lors de la tenue de l'exposition Les Arts anciens de l'Amérique, au musée des Arts décoratifs ${ }^{6}$. George Henri Rivière entrera dans les fonctions de sous-directeur du musée à la fin de l'été 1928 et ne sera titularisé dans son poste qu'en mars 1929. Les deux hommes vont procéder à la réorganisation complète du musée, qui connaît en effet une première consécration avec le retour de la mission Dakar-Djibouti en 1933, et non en 1935 (p.1381).

Enfin, Paul Rivet n'a jamais été élu député du cinquième arrondissement de Paris en 1920 contre le colonel de la Rocque, représentant des Croix de feu (p.1380). Il fut élu en mai 1935 conseiller municipal du quartier Saint-Victor à Paris, contre Georges Lebecq, un des instigateurs du 6 février 1934, sur une liste unitaire de gauche préfigurant le Front populaire de $1936^{7}$. S'il fut bien élu député, ce ne fut qu'en 1945, à la première Assemblée constituante, sur une liste SFIO.

Si André-Marcel d'Ans insiste par contre avec raison sur le caractère holiste du projet scientifique de Paul Rivet (p.1386), dans la lignée de Paul Broca et de Armand de

\footnotetext{
${ }^{5}$ Christine Laurière, « Paul Rivet, vie et œuvre », op.cit. : 118.

${ }^{6}$ Christine Laurière, «George Henri Rivière au Trocadéro. Du magasin de bric-à-brac à la sécheresse de l'étiquette », Gradhiva, 33, $2003:$ 61-62.

7 Cf. la notice de Nicole Racine, «Paul Rivet», in J. Maitron (éd.), Dictionnaire biographique du mouvement ouvrier français et Christine Laurière, « Paul Rivet, vie et œuvre », Gradhiva, 26, 1999 : 117.
} 
Quatrefages, il faut toutefois préciser qu'il ne s'agit plus vraiment du même projet. La différence de taille avec ses prédécesseurs se situe dans la hiérarchie de ces disciplines que Paul Rivet établit. L'anthropologie physique n'a plus la prééminence, car il a sévèrement raboté ses prétentions pour ne plus la mobiliser qu'en tant que simple auxiliaire de disciplines telles que la linguistique, l'ethnographie, l'archéologie. Cette inversion dans l'ordre des sciences prioritaires faillit d'ailleurs lui coûter son élection à la chaire d'anthropologie du muséum en 1928, tant les partisans d'une anthropologie dure se déchaînèrent contre sa candidature.

C'est donc à ce conglomérat de sciences que va devoir se familiariser Jacques Roumain lorsqu'il s'inscrit à l'Institut d'Ethnologie, pour l'année universitaire 1937-1938. Il suit plusieurs enseignements, dont celui de Paul Rivet sur « Les origines de l'homme américain », ce qui est attesté par un petit cahier de notes (p.1387). Vraisemblablement, il a aussi assisté aux Instructions d'anthropologie physique, qui comprenaient des leçons sur les caractères et classification des races humaines, une mise au point sur les définition de race et de peuple, une histoire des migrations mondiales et des métissages qui en ont découlé, etc. ${ }^{8}$ A n'en pas douter, ce cours l'a aidé à structurer davantage son discours sur le sort inique réservé aux populations noires et lui a fourni un arsenal d'arguments scientifiques dont il a su tirer profit dans son essai : «Griefs de l'homme noir», publié en 1939 dans l'ouvrage collectif L'Homme de couleur (pp.703-718). Il y démonte plusieurs préjugés raciaux censés motiver la ségrégation aux Etats-Unis : le caractère pernicieux du métissage, l'infériorité irréductible de la race noire, la protection de la femme blanche. Ironisant amèrement sur ces « Américains, qui

\footnotetext{
${ }^{8}$ Une phrase de Griefs de l'homme noir laisse entendre que Jacques Roumain a pu assister à ce cours : «Le professeur Rivet, dans un de ses cours au Muséum d'histoire naturelle [...]»(p.710). Cf. le cours d'anthropologie physique de Paul Rivet (archives BMH, fonds Paul Rivet, 2 AP 1 B13c).
} 
ne manqu[a]nt pas d'originalité, ont, quant à eux, inventé la notion de l'homme nordique caucasique »(p.708), Jacques Roumain cite l'ouvrage de Paul Lester et Jacques Millot, Les Races humaines, qui rappelle à l'envi que « toutes les populations sont métisses, cent fois métisses, et qu'aucune d'entre elle n'appartient tout entière à un seul groupe » (p.708). A cette époque, Paul Lester était le sous-directeur du laboratoire d'anthropologie du Muséum national d'histoire naturelle, sous la tutelle directe de Paul Rivet. Jacques Roumain convoque également ce dernier pour démontrer que le métissage n'est pas une tare, que les populations noire et blanche peuvent très bien se mélanger, comme c'est le cas au Brésil (p.710).

Le cursus dudit Institut prévoyant également un cours de muséographie et de travaux pratiques, dans les locaux du musée de l'Homme, Jacques Roumain est amené, comme tous les autres étudiants en ethnologie, à participer au classement, rangement et étiquetage des collections ethnographiques et archéologiques. Etant donnée sa nationalité, il fut probablement attaché au département Amérique, qui abritait les collections caribéennes. La nature de ces activités n’en firent pas pour autant «l'un des assistants de Paul Rivet au musée de l'Homme » (p.1218), ainsi que cela est un peu trop hâtivement avancé, mais plutôt un membre de cette «petite armée d'une cinquantaine de travailleurs auxiliaires $»^{9}$ qui, bon an mal an, se succédait au chevet d'un musée en pleine réorganisation, consacrée par l'inauguration officielle du nouveau musée de l'Homme, le 20 juin 1938.

Appartenant «à ce groupe de jeunes écrivains et poètes qui, en Haïti, se sont efforcés de créer une littérature d'expression française, mais de contenu nègre ${ }^{10}$,

\footnotetext{
9 Paul Rivet, Paul Lester et Georges-Henri Rivière, «Le laboratoire d'Anthropologie du Muséum », Archives du Muséum, 6ème série, XII, 1935 : 521.

${ }^{10}$ Texte de présentation de la radio-conférence de Jacques Roumain, « Croyances religieuses populaires d'Haïti », 9 mai 1938 (Archives de la bibliothèque du musée de l'Homme, cote : 2 AM 1 C9c). A paraître
} 
Jacques Roumain est sollicité - vraisemblablement par Jacques Soustelle, le nouveau sous-directeur du musée de l'Homme - pour prononcer une des radio-conférences dont avait la charge le musée. En effet, depuis mai 1935, chaque mardi, la tranche horaire de 18 heures-18 heures 15 de Radio-PTT puis de Radio Paris, était animée par une « causerie ethnologique $»^{11}$, dite par une personne en relation avec le musée ou l'Institut d'Ethnologie. Plus de 125 radio-conférences seront ainsi données sur les ondes jusqu'à l'été 1939. Jacques Roumain anime la $96^{\text {ème }}$, avec pour thème les «Croyances religieuses populaires d'Haïti ». Dans ce court texte, où on ne le sent pas très à l'aise pour traiter d'un thème qu'il aborde avec une certaine raideur, il insiste sur les « relations d'une intimité totale » entre le vaudou et le catholicisme populaire. «Alors que le protestantisme exerce une influence destructrice sur le vaudou, un procès d'absorption de la part de celui-ci, s'est accompli quant au catholicisme. Les divinités vaudouesques se superposent exactement à certains saints catholiques [...]». Il termine en affichant sa «plus affectueuse amitié » au paysan haitien qui, «malgré ses pratiques archaïques, est un travailleur acharné, honnête et fier »! On trouvé là énoncée toute l'ambiguïté de la pensée ethnologique de Jacques Roumain : soucieux de valoriser le petit peuple haïtien et son mode de vie populaire, par humanisme et conviction marxiste, il n'en est pas moins embarrassé de devoir évoquer cet obscurantisme religieux qui entache le drapeau national pour les élites haïtiennes. La dernière phrase de sa causerie prend alors tout son sens : il souhaite jeter une fois pour toutes les « contes à dormir debout sur les zombies-revenants, les loups-garous, les envoûtements et autres sornettes [...] au panier des et cetera », afin de normaliser le vaudou et de le débarrasser de ces représentations fantasmées .

\footnotetext{
dans Gradhiva, n.s., 1, 2005.

${ }^{11}$ L'expression est de Georges-Henri Rivière, sous-directeur du musée d'ethnographie du Trocadéro de 1928 à 1937.
} 
Inquiet des menaces de guerre pesant sur l'Europe, Jacques Roumain quitte la France fin mai 1939 et rejoint les Etats-Unis. Son souhait le plus cher est alors de poursuivre ses études d'anthropologie à l'Université de Columbia. Malheureusement, accablé de difficultés financières, il ne peut s'acquitter des droits d'inscriptions onéreux qu'exige l'université. Il parvient vraisemblablement à suivre un séminaire, voire deux, durant un semestre, entre septembre 1939 et janvier 1940. A Paul Rivet, il écrira qu'il a «poursuivi aux Etats-Unis des études d'Anthropologie ${ }^{12}$, gonflant peut-être un peu son curriculum vitae pour asseoir sa légitimité. Mais des problèmes pécuniaires mettent rapidement un terme à ses ambitions scientifiques. Son passage à La Havane entre décembre 1940 et mai 1941 lui permet néanmoins de profiter des bonnes conditions de recherches que lui offre la Bibliothèque nationale cubaine et d' « attaqu[er] à nouveau ce vieux travail sur l'ethnologie précolombienne d'Haïti » (p.1392). Celui-ci sera publié en février 1942 dans le premier numéro du Bulletin du Bureau d'Ethnologie sous le titre de «Contribution à l'étude de l'ethnobotanique précolombienne des Grandes Antilles ».

L'élection en Haïti en mai 1941 d'un nouveau président de la République, Elie Lescot, lui permet d'envisager son retour sur la terre natale et de mettre fin à un exil douloureux. Mais il n'a pas abandonné pour autant ses ambitions scientifiques, qu'il souhaite mettre dorénavant au service de son pays. Soucieux de doter Haïti de structures semblables à celles de Etats-Unis ou de la France, il a «proposé au Dr. Price-Mars, l'ethnographe haïtien, de créer ici un Institut d'Ethnologie. Le passage à Port-au-Prince de Soustelle, puis de Métraux [va] donn[er] une nouvelle impulsion à ce projet $»^{13}$, souligne-t-il. Il est certain que les nombreuses conservations entre Roumain et Métraux vont s'avérer déterminantes (p.1393-94). Les deux hommes se sont rencontrés le 17

\footnotetext{
${ }^{12}$ Lettre de Jacques Roumain à Paul Rivet, Port-au-Prince, 3 novembre 1941 (archives BMH, fonds Paul Rivet, 2 AP 1 C). A paraître dans Gradhiva, n.s., 1, 2005.

${ }^{13}$ Lettre de Jacques Roumain à Paul Rivet, op.cit.
} 
juillet 1941 chez des relations communes, les époux Lhérissson. De cinq ans plus jeune que Alfred Métraux, Jacques Roumain lui «fait fort bonne impression $»^{14}$, et ils nouent immédiatement des liens de sympathie. Les événements liés à la Renonce (nom créole donné à la campagne anti-superstitieuse) leur fournissent matière à de nombreuses discussions. Les autodafés de milliers d'objets sacrés du rituel vaudou, la destruction des sanctuaires familiaux, les menaces d'excommunication du clergé catholique, font craindre à Alfred Métraux une disparition rapide du culte. Partageant la même préoccupation, Jacques Roumain est « convaincu de la nécessité de sauver le souvenir du vaudou, si gravement menacé. De [ces] conversations naquit l'idée de créer en Haïti un "Bureau d'ethnologie", spécialement chargé de cette tâche ${ }^{15}$ de recueillir pendant qu'il en était encore temps des objets vaudou. Il publiera un article d'hommage à « Jacques Roumain, archéologue et ethnographe », publié dans les Cahiers d'Haïti en novembre 1944 quelques mois après sa mort (reproduit pp.1633-1636), où il revient sur leur dialogue et évoque l'institutionnalisation de l'ethnologie en Haïti, saluant les réalisations de son $\mathrm{ami}^{16}$.

Un Institut et un Bureau d'Ethnologie verront ainsi le jour à l'automne 1941, le premier dirigé par le docteur et folkloriste Jean Price-Mars, le second par Jacques Roumain. Le tout nouvel Institut d'Ethnologie ouvre ses portes en novembre 1941, avec un programme calqué pour partie sur celui de son aîné parisien. Il a orienté ses activités vers l'enseignement et la formation d'ethnologues. Promu professeur malgré son faible bagage universitaire, Jacques Roumain y dispense un cours d'anthropologie

\footnotetext{
${ }^{14}$ Alfred Métraux, Itinéraires 1 (1935-1953). Carnets de notes et journaux de voyage, compilation, introduction et notes par André-Marcel d'Ans, Paris, Payot, 1978 : 133.

${ }^{15}$ Alfred Métraux, Le vaudou haïtien, Paris, Gallimard, 1998 [1958] : 13.

${ }^{16}$ Sur les séjours et travaux d'Alfred Métraux en Haïti, cf. Christine Laurière, «D'une île à l'autre. Alfred Métraux en Haïti », Gradhiva, n.s., 1, 2005.
} 
préhistorique et un autre sur l'ethnologie précolombienne d'Haïti. Sitôt sa création, il écrit à Paul Rivet, réfugié à Bogota depuis mai 1941, pour le prévenir et lui proposer d'instaurer des rapports scientifiques avec l'Institut d'Ethnologie que Paul Rivet vient lui-même de fonder dans cette ville en juin 1941. Il lui annonce également la fondation d'un Bureau d'Ethnologie, qu'il dirigera jusqu'à sa mort. Le Bureau fait figure de centre de recherches, et l'une de ses tâches principales est de favoriser le création d'un musée. Ainsi que Jacques Roumain le résume pour Paul Rivet, «ce bureau se propose : l'organisation d'un Musée d'Archéologie ; l'inventaire, le classement et la conservation des pièces ethnographiques et archéologiques, l'investigation méthodique et la protection des sites ; la publication d'un Bulletin comportant le résultat des recherches du Bureau et les travaux d'ethnologues haïtiens et étrangers ${ }^{17}$. Alfred Métraux racontait que l'écrivain haïtien « avait souvent déploré l'absence de Musée à Port-au-Prince qui, d'une part, sauverait de la destruction et de la dispersion les restes de la belle civilisation Arawak et de l'autre offrirait aux visiteurs une vision non seulement du passé, mais du présent de l'Ile. Il souhaitait y inclure les arts et métiers populaires et les objets des vieux cultes qui se célébrèrent dans les campagnes. ${ }^{18}$ Ce musée sera créé en même temps que l'Institut d'Ethnologie, le 31 octobre 1941. Bien que les attributions de chaque institution semblent clairement délimitées, des disputes ne vont pas tarder à éclore entre l'Institut et le Bureau, chacune étant jalouse de ses prérogatives - Alfred Métraux en prendra pleinement conscience lorsque lui-même aura des responsabilités officielles au sein de l'Unesco en 1948, et qu'il devra traiter chacune sur le même pied, sous peine de compromettre son travail en Haïti ${ }^{19}$.

\footnotetext{
${ }^{17}$ Lettre de Jacques Roumain à Paul Rivet, Port-au-Prince, le 3 novembre 1941, op.cit. .

18 Alfred Métraux, « Jacques Roumain, archéologue et ethnographe », op.cit. : 1636.

${ }^{19}$ «In the last years, the local «anthropologists» have fallen apart and formed two warring clans : the Bureau d'Ethnologie and the Institut d'Ethnologie without speaking of minor factions. My problem was to
} 
Jacques Roumain s'est illustré sur un autre terrain, où on l'aurait moins attendu étant données ses convictions communistes, celui de la religion et du vaudou. En pleine Renonce, il a indéniablement joué un rôle majeur dans la neutralisation de la vindicte cléricale. Son pamphlet, « A propos de la campagne "anti-superstitieuse” », paru entre les 11 et 18 mars 1942 dans le Nouvelliste (reproduit pp.745-752), le projette en première ligne pour affronter le clergé catholique haïtien et le pousser dans ses derniers retranchements idéologiques et politiques. Réfutant catégoriquement le qualificatif de «superstitieux » pour caractériser le vaudou, qui serait plutôt un culte syncrétique, Jacques Roumain accuse le «clergé concordataire » d'impéritie, l'estimant coupable de la «retentissante faillite de l'évangélisation catholique »(p.749). Faussement naï, il affirme que le clergé ne peut s'en prendre qu'à lui-même, puisque «la persistance d'un des facteurs - le vaudou - dépend de l'existence de l'autre : le catholicisme. [...] Ce que l'on peut reprocher au Clergé, c'est d'avoir laissé des prêtres ignorants offrir à nos masses une vision si élémentaire du surnaturel qu'une fusion des croyances africaines et catholiques a pu se réaliser. »(pp.749-750) Et puis, ce n'est pas d'une campagne anti-superstitieuse dont ont besoin les paysans, mais d'une «campagne anti-misère » (p.751) : seules l'amélioration des conditions matérielles et sanitaires, la dispense de soins médicaux, l'éducation, permettront aux Haïtiens de s'affranchir du culte des loas, pense un Jacques Roumain confiant dans le progrès matériel. A «conditions économiques égales ", le paysan haïtien n'est en effet pas plus superstitieux que le paysan français (p.749). Ce n'est assurément pas un plaidoyer en faveur du vaudou qu'il prononce : ce culte s'éteindra de lui-même lorsque les masses accéderont au progrès, 61). 
deviendront des citoyens à part entière d'un état moderne, et il n'y aura pas lieu de le regretter, car le vaudou freine leur émancipation et les maintient dans la peur superstitieuse.

Mais selon André-Marcel d'Ans (pp.1400-1418), le pamphlet de Jacques Roumain obéit à des motivations bien moins intellectuelles et scientifiques que politiques. Il aurait ainsi fait le jeu du président Elie Lescot, défavorable à l'outrance et aux égarements de prêtres mettant en danger la concorde civile. Il y aurait alors eu une espèce de collusion objective entre les intérêts de Lescot, catholique fervent mais désireux de protéger ses prérogatives et son autorité, et ceux de Jacques Roumain, de conviction marxiste, mais, dans le cas présent, patriote avant tout. L'immixtion de prélats bretons dans les affaires haïtiennes, en ces temps de guerre mondiale où Haïti a choisi le camp des Alliés, est assimilée à une ingérence insupportable de la part d'une « hiérarchie française $[\ldots]$ pro-Vichy, pro-collaborationniste, $[\ldots]$ anti-alliés » et, finalement, «pro-fasciste » (p.752). Face à cette hostilité croissante, la campagne anti-superstitieuse cesse d'elle-même et les sanctuaires vaudou rouvrent progressivement.

En septembre 1942, Jacques Roumain est nommé par le gouvernement haïtien chargé d'affaires d'Haïti à Mexico. Il y à là de quoi s'étonner, si l'on garde en mémoire le parcours politique de Roumain. René Depestre va jusqu’à parler d'un «"mystère” existentiel. Pourquoi le principal disciple haïtien de Marx a-t-il accepté de représenter au Mexique le régime dictatorial que ses proches amis continuent à harceler dans un quotidien de Port-au-Prince?»(p.XXIX) Exil doré imposé par un gouvernement soupçonneux pour certains (p.1223), obéissance à la ligne du Komintern qui prônait l'obéissance aux régimes alliés, même dictatoriaux (p.XXIX), ou bien encore 
récompense à un allié un peu trop encombrant pour services rendus dans la lutte contre un clergé envahissant pour d'autres p(.1418), l'énigme reste entière. Peut-être, tout compte fait, est-ce René Depestre qui a raison quand il rappelle que Jacques Roumain était malade, et qu'il a peut-être voulu trouver un peu de repos auprès d'amis, et se recentrer sur l'écriture. Le fait est qu'il n'abandonne ni l'ethnologie ni la littérature, écrivant deux textes majeurs, Le sacrifice du tambour Assôtô( $(r)$ et Gouverneurs de la rosée. Du premier texte, Alfred Métraux écrivit que : «pour la première fois nous avons un rite sacrificiel de la religion populaire haïtienne décrit avec un luxe de détails et une clarté qui un font un modèle de monographie ethnographique.» (p.1635) André-Marcel d'Ans invite à relativiser ce propos, énoncé dans le cadre formaté de l'hommage post-mortem, et à relire le texte sans parti pris. Le fait est qu' « il s'agit d'une œuvre bizarre, énigmatique, à la fois riche d'informations mais difficilement lisible » (p.1419), au plan décousu, et qui fait référence à des aires culturelles incroyablement diverses. Il n'est pas sûr que Jacques Roumain ait observé une telle cérémonie, se reposant sur un seul informateur, le prêtre vaudou Abraham, qu'Alfred Métraux lui-même rencontrera plus tard. Il y a quantité d'informations, de chants, manque la méthode ethnographique qui ordonnerait le tout. Il semble également que Jacques Roumain ait été plus intéressé par l'archéologie - il lui consacre deux articles et l'essentiel du temps qu'il passe en Haïti entre la fin de son exil et son départ pour Mexico- que par l'ethnologie, pour laquelle il avait moins d'affinités et dont il avait moins la pratique. Homme de sa caste, Jacques Roumain ignorait à peu près tout de la vie paysanne, ce que n'a pas manqué de relever André-Marcel d'Ans dans sa lecture orientée - ethnologique, et non pas littéraire - de Gouverneurs de la rosée qui, relève-t-il, «ne concerne plus qu'une paysannerie abstraite, esthétisée, ethnologiquemement fantasmée. » (p.1424) Il n'en reste pas moins que faire surgir dans 
l'arène littéraire haïtienne cette figure du paysan, même travaillée par la fiction, victime et héros tout à la fois, relève tout autant du coup de force symbolique que de l'acte politique.

C'est ainsi qu'il faut interpréter l'un de ses tout derniers engagements publics en faveur de la connaissance scientifique des populations noires en Amérique. Mi-février 1944, Paul Rivet reçoit une lettre du tout nouvel Institut international des études afro-américaines, l'informant de sa création le 20 octobre 1943 à Mexico, et lui proposant de bien vouloir faire partie des membres fondateurs. Ses animateurs sont les anthropologues cubain Fernando Ortiz et mexicain Gonzalo Aguirre Beltrán (qui s'illustreront dans ces études pionnières), Jaques Roumain et Daniel Rubín de la Borbolla. Ils veulent fédérer et susciter un pôle d'études sur les Noirs du continent américain, «dans leur aspects biologique et culturel, et de leur influence sur les peuples américains $»^{20}$. « Il n'est pas nécessaire d'insister sur la signification d'un groupement comme celui-ci ni d'en justifier l'existence », précise la lettre. L'Institut se propose de mener des recherches et d'informer les gouvernements des résultats de ces enquêtes. C'est un champ d'études en sciences sociales relativement inexploré qui s'offre à eux, et il est significatif que Jacques Roumain, dépassant la question noire haïtienne, se joigne à un collège international souhaitant examiner de manière dépassionnée ces problèmes cruciaux dans la représentation symbolique que se font d'elles-mêmes les nations américaines.

Christine Laurière

\footnotetext{
${ }^{20}$ Lettre du 15 février 1944, archives BMH, fonds Paul Rivet, 2 AP 1 C. A paraître dans Gradhiva, n.s., 1, 2005 .
} 